\title{
Structured Model Representation of Manufacturing Processes Using Petri Nets and Knowledge Based Tools
}

\section{Horváth}

Department of Manufacturing Engineering, Bánki Donát

Polytechnic Népszinház u. 8. Budapest H-1081 Hungary

phone: (36-1)-210-1449, fax: (36-1)-133-6761

e-mail:lhorvath@zeus.banki.hu

\section{I.J. Rudas}

Department of Information Technology, Bánki Donát Polytechnic

Népszinház u. 8. Budapest H-1081 Hungary

phone: (36-1)-133-4513, fax: (36-1)-133-9183

e-mail: rudas@zeus.banki.hu

\section{M. Camarinha-Matos}

New University of Lisbon Quinta da Torre 2825 Monte Caparica, Portugal phone: (351-1)-3500224, fax: (351-1)-2941253 e-mail: cam@uninova.pt

\begin{abstract}
In this paper an approach to manufacturing processes modeling and knowledge based decision making at manufacturing process planning is proposed. The main objective is to develop advanced decision support software tools for CAD/CAPP/CAM and flexible manufacturing systems. Earlier experiences of authors revealed characteristics of decision making at manufacturing process planning. Manufacturing processes modeling by process features is proposed. Integration of process planning with production scheduling in a common knowledge based decision making environment was promoted by application of non-linear process models. Petri net formalism has been chosen for representating process features. Process features are attached to feature based model entities of mechanical parts. For product model the STEP approach is followed. Finally, the knowledge representation and evaluation of the models during manufacturing process planning are outlined.
\end{abstract}




\section{INTRODUCTION}

In recent years professional CAD/CAM and flexible manufacturing systems have gained wide acceptance in the industry. Importance of the small batch production is growing in these days. This causes constantly increasing role of powerful CAD/CAM techniques and flexible manufacturing systems (FMS). Manufacturing process planning (CAPP) procedures that can be available for creating process plans in compliance with actual demands at any time make possible the utilization of flexibility of an FMS. In order to cope with breakdowns, capacity bottlenecks and changes in the production schedule it is necessary to create new process plan variants. This assumes reliable knowledge based CAPP decision making tools and, as a consequence, the importance of developing methodologies for manufacturing process modeling can be recognized. The product model approach for organizing the manufacturing information system also raised the issue of manufacturing process modeling. Geometric modeling, as supported by tradittional CAD systems, ensures sophisticated computer representation of shapes. At the same time there are not appropriate manufacturing process model representations for product models.

In the last decade research efforts brought some experiences at adaptation of advanced problem solving methodologies in manufacturing process planning. Most of the researchers are aimed at development of stand-alone manufacturing process planning systems. The authors of this paper are working on development of manufacturing process planning methodology with the aim of producing problem solving tool kits for professional CAD/CAM systems and FMSs. Hungarian researchers have produced conventional manufacturing process planning systems [4]. Attempts were made for computer assisted decision making. The GLEDA system [5] is an example for these efforts. This system has been successfully implemented at various company environments in Hungary. On the basis of experience gained at that project, a new process modeling and planning approach has been evolving to utilizing the new problem solving procedures. On the other hand, the New University of Lisbon has large experience on CIM information systems, integrating infrastructures and interactive decision making / planning systems for CIM. The EIMS/CIMFACE [15],[16], and CIM-CASE prototypes [17] are examples of such activity. The cooperation between the two groups aims at establishing an integrated approach for interactive process planning and modeling. This paper summarizes the proposed approach and introduces some methodology.

Petri nets, fuzzy logic and other knowledge acquisition and representation tools have been applied for creating manufacturing process models. In particular, a special interpretation of high level Petri net formalism together with the object oriented paradigm has been involved for modeling of manufacturing processes. The production scheduling calls for process plan variants that are conformant with all technological constraints. For that reason the proved concept of the non-linear process plan has been adapted [13], [8].

Application of conventional knowledge based manufacturing process planning methods has resulted in software tools that are not well suited to manage decision making in present day $\mathrm{CAD} / \mathrm{CAM}$ systems. Separated decision making functions which can be attached to the process modeling functions of commercial CAD/CAM systems and may be used by the human according to his judgment are required. The manufacturing engineer will make use of decision support features of the process planning system according to the nature of the task. The human is responsible for the entire design and planning work so that he must be able to supervise the decision support procedures in a balanced way.

The paper is organized as follows. First, the problem and current emphasis on manufacturing process modeling are explained. Then the proposed manufacturing process model is outlined. Following this the Petri net model representation is detailed. Finally, the knowledge representations and evaluation of the models during manufacturing process planning are outlined.

\section{PROBLEMS AND CURRENT EMPHASIS}

Numerous research and development projects have been initiated during the last two decades. At the earlier eighties the first generation of expert systems offered the promise of advanced 
solutions. Researchers tried to implement rule /frame based hybrid expert systems for CAPP. Most of these CAPP expert systems utilized backward chaining techniques [11]. In these procedures the reasoning process starts from the finished part and seeks for a manufacturing process element (manufacturing step or operation) that results in the finished state. Omitting the influence made by that manufacturing process element on the workpiece, a "less-machined" workpiece is defined. Then a new process element is sought which results in this less-machined workpiece. The reasoning process is continued until the less machined workpiece is the blank (raw material). These systems are based on incomplete process models. At the same time these expert systems involve knowledge bases, which are complicated, special and in some cases hardly understandable for a production engineer.

A variety of CAPP methods have been proposed over the past twenty-five years. On the basis of earlier research on conventional CAPP systems [12] the development of the GLEDA process planning system at late eighties resulted in comprehensive knowledge storage and retrieval possibilities and improved user interface [15]. The experiences gained with the GLEDA system have been utilized by the authors at their recent work.

A variety of robust CAD/CAM systems are available in these days. They are becoming more and more important for the creative design and planning practice. The computer aided manufacturing process planning (CAPP) is often referred to as a missing link between the CAD and CAM or production scheduling. This is because the process planning is a very complex, decision intensive and heterogeneous activity and calls for a special methodology differing from that used by the commercial CAD/CAM systems. The processing of large amount of information demands less or more decision support. Excellent tools have been developed for solving geometric processing tasks. For that reason commercial CAD/CAM systems involve CAPP functions mainly for geometric processing.

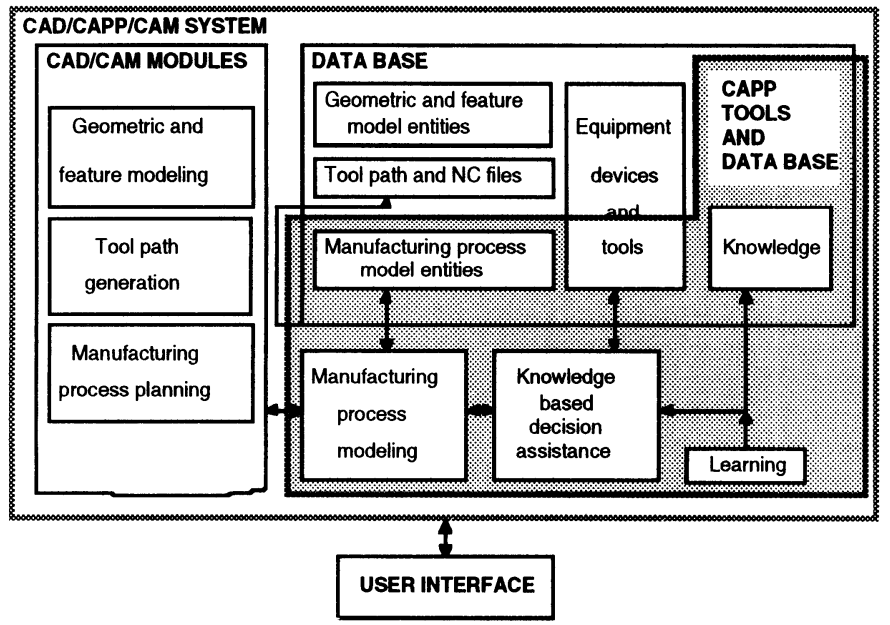

Figure 1 CAPP modeling and problem solving system elements in a CAD/CAPP/CAM system

This work aims the introduction of knowledge based methodologies and procedures for process planning activities that are not available in present day CAD/CAM systems (Fig. 1). An important factor is the feasibility of the development in terms of the programming environments of these systems. The decision support and problem solving methodology, as well as the representation of the process model are investigated taking into account the demands of novel design, planning and manufacturing systems. Lack of standard models and the large number of model representations in commercial product modeling systems give rise to difficulties. The STEP standard, once widely adopted, can give a solution for this problem [12]. 
The general structure of a professional CAD/CAM system is visualized in Figure 1. The basic structure has been enlarged with CAPP modeling, problem solving and data base elements. The new elements can be placed in the system as user defined procedures and data sets. Learning procedures may be involved [3]. A previous work of the authors have focused on acquisition and representation of manufacturing process planning knowledge [6] and manufacturing process execution [14].

\section{MANUFACTURING PROCESS MODEL IN A PRODUCT MODEL}

The product model concept implies a manufacturing process model representation that can be integrated with other partial models. The manufacturing process model can be considered as a partial model of a product model. Figure 2 shows some of the most important partial product models and modeling procedures that are related to the manufacturing process model and modeling. The most important partial models in this context are the geometrical, the technological, the feature and the manufacturing process model [1]. The technological model involves tolerances, surface properties and other technological data of the product. Production schedule is in close connection with this modeling environment in terms of the assigned types of manufacturing resources.

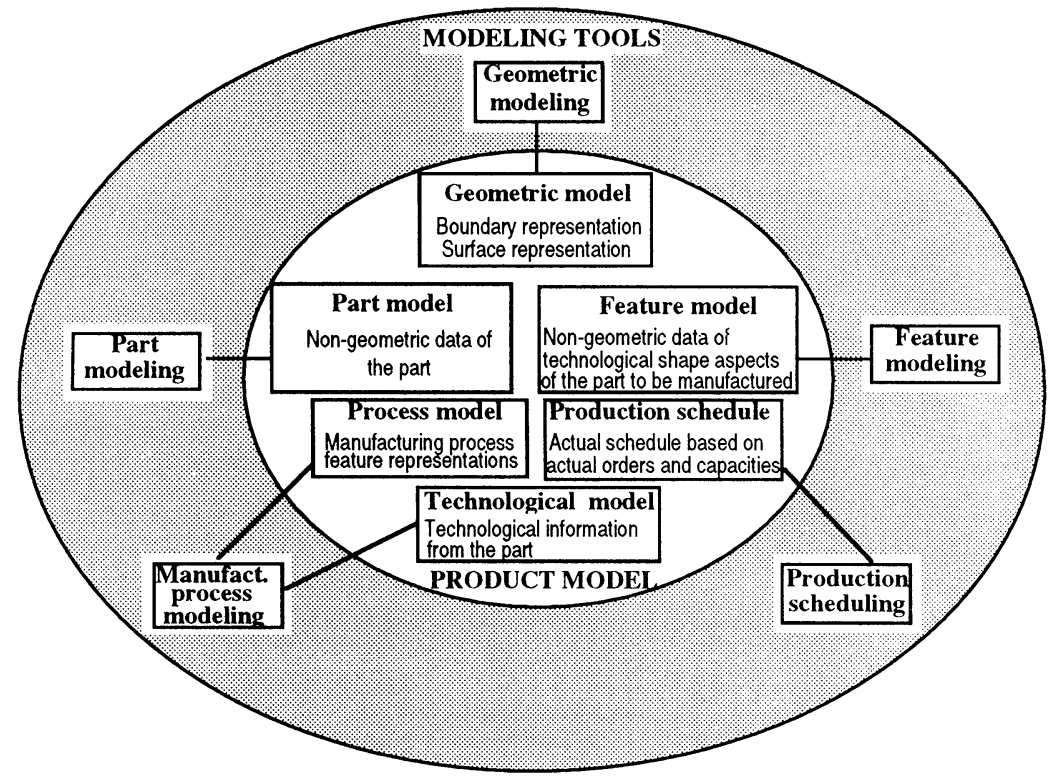

Figure 2 Manufacturing process modeling in the framework of the product model concept

Recently, the feature based approach is wide spreading in the part modeling. The features make representation of non-geometric attributes possible. It is logical to define similar entities for the manufacturing process so that similar principles may be applied for part and process modeling. Nowadays only a few of the advanced CAD/CAM systems offer functions for creation and management of manufacturing process entities [10], [12]. An example is the EUCLID3 system. The manufacturing process is handled as an entity that represents the place of "sequences", "operations" and "cycles" in a tree structure. A process is a series of "sequences". A "sequence" is a series of operations performed by a given machine tool with a given set-up. An "operation" is a 
succession of machining cycles performed by the same tool. Material to remove is an additional entity for process planning. An objective at the development of the PRODES system [10] was the integration of the process planning with the product modeling. The process entities in this system are called "process features" that are related to the "form features" of the part model. The PRODES system uses special process features for description of manufacturing sub processes attached to each of the features. This is based on a proved principle [4].

The [12] proposes process sequence, process, operation and path features. The "process" in this concept represents operations that are performed on the same form feature using the same manufacturing process (e. g. milling). The manufacturing of a part is represented by the process sequence feature.

\section{APPROACH TO MANUFACTURING PROCESSES MODELING}

The human manufacturing process planner first conceptualizes a process then elaborates subprocesses for the features, finally places the subprocesses into the process concept. Modeling of the manufacturing process follows this logic. The manufacturing process is represented as a sequence of setups. The setup is defined as a sequence of operations that take place at a given setup position of the workpiece on a given machine. The segment and the feature subprocess involve operations. Operation refers to a machining action that is done by a given tool on one or more surfaces. The feature subprocess, the setup and the operation are manufacturing process features to be established and parametrized at the various levels of process modeling. In the case of CNC the operation has a pointer to machining cycles. This approach has been developed for parts that are manufactured by machining, but it can be adapted to other manufacturing processes.

The proposed process model has a four-level structure (Figure 3). Features on a higher lever are related to features on lower levels. The features of the process model are attached to a process, to manufacturing of a part or a form feature as well as to manufacturing process components such as a setup or a specific operation sequence. The process features are modeled as generic manufacturing process components. Representation of a process component contains information on all available process variants and knowledge for the evaluation procedure. A given process feature is suitable for a cluster of mechanical parts, form features or setups. Well-structured information is involved in the data base for equipment, tools and fixtures that are available in a factory environment. The generic process model components are represented using extended object oriented Petri net formalism, taking into account the demands of both process planning and production scheduling. A Petri net model represents all process variants that are suitable for a cluster of manufacturing tasks. Rules or rule sets can be attached to the place elements of the Petri nets. Rule objects are processed using special knowledge based problem solving methods. Uncertainties that are usual in process modeling can be handled by fuzzy reasoning [2].

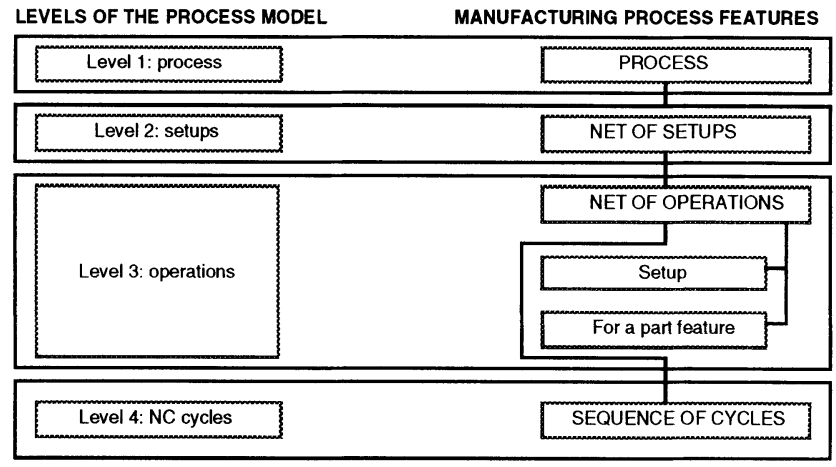

Figure 3 Levels of the process model 
Manufacturing process features are placed on the process, the setups, the operations and the cycles level of the process model (Figure 3). Process features are included in the Petri net models as transitions. Each transition points to another Petri net model on the next lower level of the manufacturing process model. The process is a hierarchy of process features except for the net of operations for a feature manufacturing.

The part model has attributes such as feature types, overall configuration, lot size, dimensions and qualitative specification. The generic manufacturing process feature is selected on the basis of values of these attributes. When the process is decided, the appropriate procedure is retrieve, and checked. When several process objects are proved to be suitable, a final selection step is encountered. A net of setups feature is attached to the manufacturing process feature. Selection of a net of operations process object for a manufacturing feature is done in similar way. The term "manufacturing feature" is for application feature that points to a form feature as defined according to the FFIM of the STEP. The above mentioned process features describe manufacturing processes of technical model features of a part. The geometric model representation of the part is attached to the part and the form feature models. The cycle feature has a specific characteristic. It is attached to geometric model object.

A set of knowledge representation tools are used for structured and unstructured knowledge (Figure 4.). Five main groups of rules are considered: rules for process selection, rules for feature manufacturing process selection, rules for nets of setups, rules for nets of operations for setups and rules for nets of operations for features. Selection of process and feature manufacturing process are done using classification trees. These trees are generated by appropriate clustering procedures. Background knowledge contains information about objects.

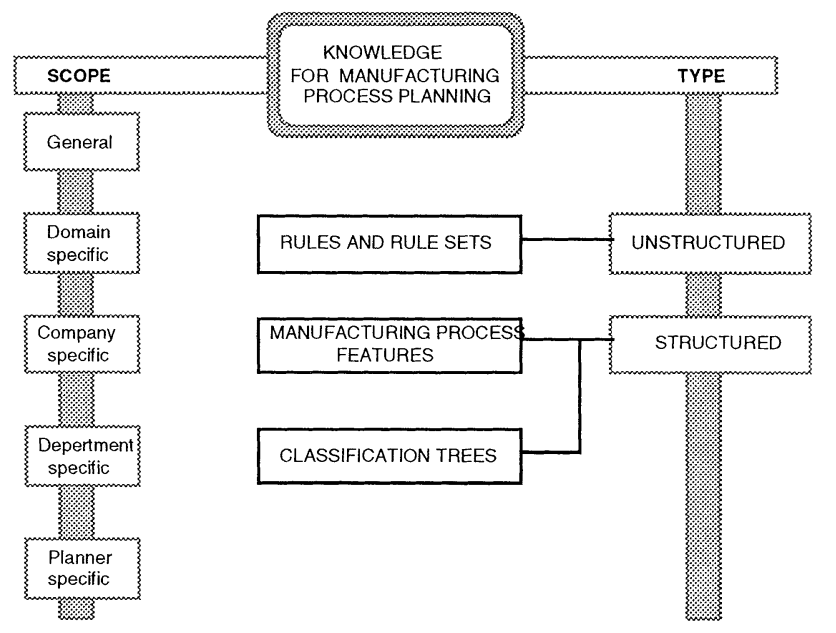

Figure 4 Characteristics of the process planning knowledge

\section{PETRI NETS REPRESENTATION OF MANUFACTURING PROCESS FEATURES}

IF-THEN rules are attached to places and used at the evaluation of the Petri net. Rules are assigned to a place type or a place instance. Rules assigned to a selection procedure or a place may 
contradict each other as usual at manufacturing knowledge. Contradicting rules are processed using Fuzzy logic into a resultant IF-THEN rule. Special transitions are for creating branches. These transitions are OR and AND splits and joins. Firing conflicts are avoided in this manner. Uncertainties at selection amongst branches are handled by processing fuzzy rules placed at the appropriate places. These groups of places are enclosed by dashed line in Fig. 5. There are rules in a Petri net model that relate places. These relations describe for example interdependence between decisions at places. If a selection between branches is to be made, the places after the or split are analyzed together. Objects define manufacturing process features, Petri net components and rules [7], [9]. Object classes are created accordingly. The structure of an object class is described by means of a set of attributes, related objects and inheritance. Attaching special methods to these objects requires additional investigation.

Petri net objects are Petri net, transition, place and token. Special objects are rule and rule sets. Procedures for manufacturing process model generation create instances of these objects. The process model for a part is constituted by these instances. Place instances carry rules. This structure of object is undergone the evaluation procedure. Typical Petri net model examples are illustrated in Figure 5.

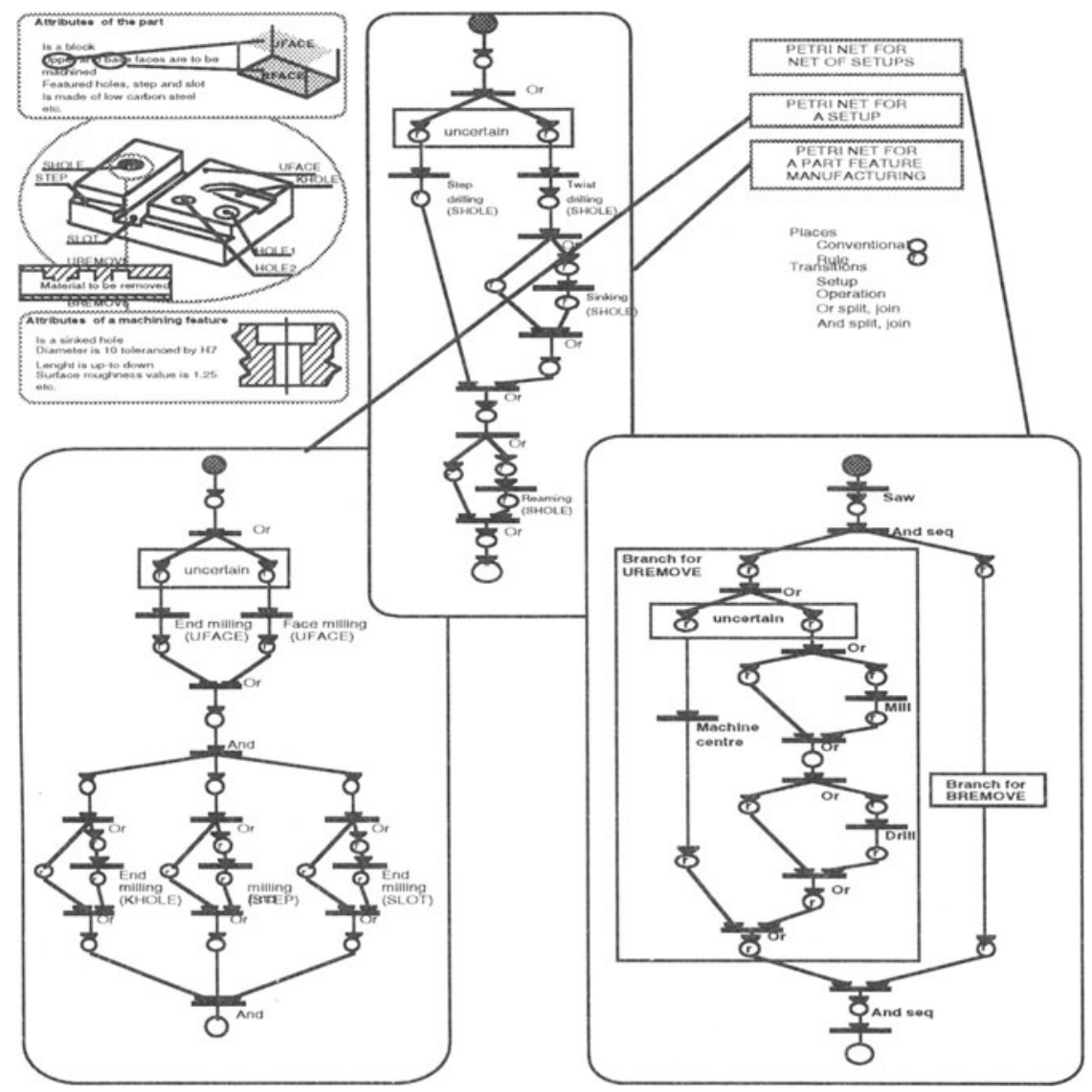

Figure 5 Examples of Petri net models 


\section{EVALUATION OF THE PETRI NET MODELS}

The evaluation of a Petri net results in a partly or an entirely evaluated net. The partly evaluated net has places with information on the results of the first (technological) evaluation stage. A partly evaluated net represents a set of process variants and requires additional evaluation to achieve a single process. The second stage of evaluation takes place at the production scheduling. The two stages can be done simultaneously using the appropriate evaluation procedure.

At the evaluation of a Petri net rule based reasoning, conventional planning procedures and dedicated Petri net evaluation procedures are available (Fig. 6). Standard evaluation procedures of Petri net handling tools must be completed by domain specific procedures.

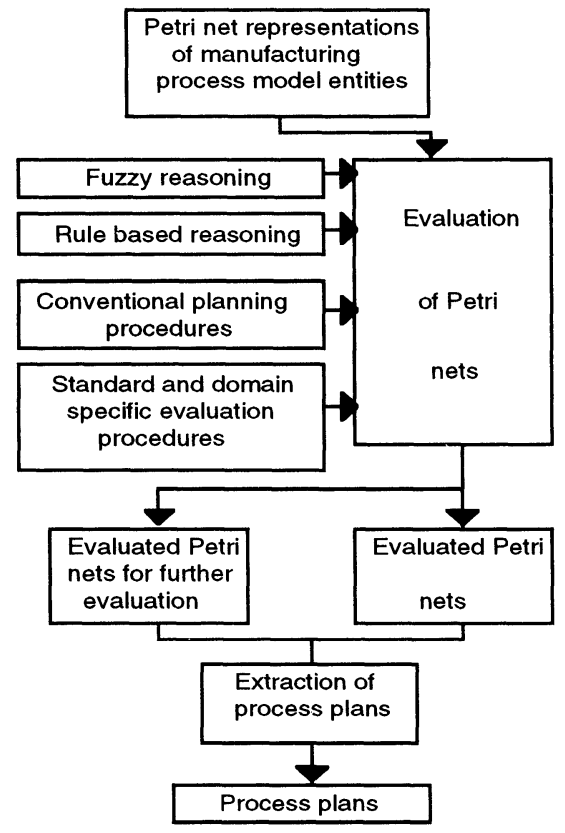

Figure 6 Evaluation of Petri nets

Evaluation of the Petri net is done by firing the transitions. Rule element makes a subsequent branch valid if attribute values are as in the IF predicate of the rule. Tokens are assigned at the usual way to the places. In course of evaluation of a Petri net, the number, type, state and position of tokens define the execution state. Transitions may be featured by priorities to help handling of firing conflicts.

\section{CONCLUSIONS}

An approach and methodology for decisive manufacturing process planning were presented. The aim was to produce a methodological base of development of software tools to support decisions made by the manufacturing engineer. Process planning, due to the large interactions with other areas (concurrent engineering perspective) has to be approached through a good balance between automatic planning functionalities and human based decision making. A feature based manufacturing process modeling approach was proposed. Four levels of the model were defined. 
Object oriented Petri nets were introduced as internal representation of complex objects emerged in a manufacturing process. The results can be utilized for the development of decisive manufacturing process planning procedures for integrated CAD/CAPP/CAM and flexible manufacturing systems. There are on-going and planned projects that use this approach and methodology in more or less extent.

\section{Acknowledgements}

We thank the Hungarian-Portuguese intergovernmantal cooperation programme for partial support to this work.

\section{REFERENCES}

1. H. Grabowski - R. Anderi - M. J. Pratt: Advanced Modelling for CAD/CAM systems, Springer Verlag, 1991

2. Zadeh, L., A.: Plenar Paper presented during IEEE International Workshop on Neuro-Fuzy Control, Japan, March 22-23, 1993

3. Kondratoff, Y. - Michalski, R.S. : Machine Learning: An Artificial Intelligence Approach, Morgan Kaufmann, 1990.

4. Horvath, M.: Computer aided manufacturing planning, new methods and applications, 3 emes JST Production Automatisee Conference, Toulouse, 1981.

5. Horváth, L.: The GLEDA computer-aided manufacturing process planning system; Intertechno '90 Conference, Budapest 1990, pp. 157-168.

6. Horváth, L. - Rudas, I. J.: Investigations on Application Possibilities of Machine Learning in Manufacturing Process Planning. Ninth International Conference on CAD/CAM, Robotics, and Factories of the Future, (9th CARS \& FOF), confer. proceedings, Newark (New Jersey, USA), 93

7. Laszlo Horvath - Imre J. Rudas: Manufacturing Process Planning using Object Oriented Petri Nets Supported by Fuzzy Reasoning. Proceedings of the 10th ISPE/IFAC Inter. Conf. on CAD/CAM, Robotics and Factories of the Future, CARs and FOF' 94, Ottawa, Canada, 1994.

8. Laszlo Horvath - Imre J. Rudas: Manufacturing Process Planning Using Object Oriented Petri Nets Supported by Entropy Based Fuzzy Reasoning. Integrated Systems Engineering, PERGAMON, Oxford, 1994, pp. 335-340.

9. Laszlo Horvath - Imre J. Rudas: Fuzzy Supported Object Oriented Petri Nets at Various Levels of Manufacturing Process Planning. Proceedings of the 1994 IEEE Int. Conf. on Systems, Man and Cybernetics, IEEE Systems, Man and Cybernetics Society, San Antonio, 1994.

10. Horváth, L., Horváth, I., Dorozsmai, K., Gábor, Zs.: Feature-Object-Based Manufacturing Process Planning with the PRODES system. IEEE International Conference on Systems, Man and Cybernetics (SMC'93) conference proceedings, Volume 4, Lille (France), pp. 21-26

11. Erve, A. H. - Kals, H. J. J.: XPLANE, a Generative Computer Aided Process Planning System for Part Manufacturing, Annals of the CIRP, 1986/1., pp. 325 - 329

12. Eversheim, W. - Marczinski, G. - Cremer, R.: Structured Modeling of Manufacturing Processes as NC-Data Preparation, Annals of the CIRP, 1991/1.. pp. 429 - 432

13 Kruth, J. P. - Detand, J: A CAPP System for Non-linear Process Plans, Annals of the CIRP, 1992/1. pp. 489-492

14 L. Seabra Lopes, L.M. Camarinha-Matos: Inductive generation of diagnostic knowledge for autonomous assembly, IEEE Robotics and Automation, Nagoya, Japan 21-27 May 95.

15 L.M. Camarinha-Matos, A.L. Osório: CIM-FACE: a federated architecture for Concurrent Engineering, in Computer Applications in Production Engineering, Proceedings of CAPE'95, Chapman \& Hall, Q. Sun, Z. Tang, Y. Zhang (Eds.), May 1995.

16 J. Barata, L.M. Camarinha-Matos: Integration of Object Oriented Programming and Petri Nets for Modelling and Supervision of FMS/FAS, R. Carelli, W. Colombo, in Balanced Automation Systems, Proceedings of BASYS'95, Chapman \& Hall, L.M. Camarinha-Matos, H. Afsarmanesh (Eds.), July 1995.

17 L.M. Camarinha-Matos; H.J. Pinheiro-Pita: Interactive planning in CIM-CASE, Proceedings of IEEE Int. Conference on Robotics and Automation, Atlanta, USA, 2-7 May 1993. 